\title{
Avaliação da eficácia do vermífugo albendazol à $10 \%$ através da análise de coproparasitológico e hemograma em bezerros da raça Nelore na cidade de Buritis,
}

\section{Rondônia}

\author{
Evaluation of the efficacy of $10 \%$ albendazole vermifuge through the analysis of coproparasitology \\ and blood count in Nellore calves in the city of Buritis, Rondônia
}

Evaluación de la eficacia de la vermífuga de albendazol al $10 \%$ mediante el análisis de coproparasitología y hemograma en terneros Nellore de la ciudad de Buritis, Rondônia

Recebido: 30/07/2021 | Revisado: 03/08/2021 | Aceito: 13/12/2021 | Publicado: 21/12/2021

\author{
Thiago Vaz Lopes \\ ORCID https://orcid.org/0000-0002-3192-1908 \\ Universidade Federal do Acre, Brasil \\ E-mail: thiagovlopes@ hotmail.com \\ João Gustavo da Silva Garcia de Souza \\ ORCID https://orcid.org/0000-0002-5899-2490 \\ Centro Universitário Aparício Carvalho, Brasil \\ E-mail: joaogarciadesouza27@gmail.com \\ Selton Gomes Maifredi \\ ORCID https://orcid.org/0000-0002-3020-6945 \\ Centro Universitário Aparício Carvalho, Brasil \\ E-mail: seltonmaifredi@hotmail.com \\ Thaís de Almeida Souza \\ ORCID https://orcid.org/0000-0002-2722-9601 \\ Centro Universitário Aparício Carvalho, Brasil \\ E-mail: thaís_medvet@hotmail.com \\ Sandro de Vargas Schons \\ ORCID https://orcid.org/0000-0001-9811-5356 \\ Universidade Federal de Rondônia, Brasil \\ E-mail: sandroschons@unir.br \\ Fernando Andrade Souza \\ ORCID https://orcid.org/0000-0002-9474-9404 \\ Universidade Federal do Paraná, Brasil \\ E-mail: femedvet@yahoo.com.br
}

\begin{abstract}
Resumo
Sendo uma das principais atividades dentro do estado de Rondônia e do país, a pecuária tem enorme importância econômica. Um dos pontos mais danoso para a bovinocultura está em torno da endoparasitose em terneiros no seu início de vida, fazendo com que esses animais tenham uma redução no ganho de peso. Apresentar a eficácia do vermífugo albendazol, através da análise de coproparasitológico e hemograma em bezerros nelore foi o objetivo do presente trabalho. Demonstrando de modo prático demonstrativo em 30 bezerros entre 2 a 3 meses, de vida em um período de 15 dias divididos igualitariamente em: Grupo I, controle, e Grupo II, tratamento, e, por final, estipular uma média final de ovos por grama de fezes para avaliar a eficiência da everminação com Albendazol à 10\% via oral. Foram realizados hemogramas e coproparasitológicos, para evidenciar o estado hematológico dos animais e Ovos por Grama de Fezes (OPG). Para se chegar ao resultado da eficácia, a fórmula utilizada foi o cálculo de Redução da Contagem de Ovos por Gramas de Fezes. Assim, a eficácia do vermífugo Albendazol à 10\% em bezerros entre 2 a 3 meses, foi de 97,51\%. Sendo assim, considerado eficaz, de acordo com o regulamento técnico do Grupo Mercado Comum (GMC). No contexto hematológico, foi observado no décimo quinto dia, relação entre uma média de eosinófilos maior nos hemogramas dos animais do grupo controle (alta carga parasitária), comparados com os animais everminados (baixa carga parasitária).

Palavras-chave: Albendazol; Bezerros; Coproparasitológico; Rondônia.
\end{abstract}

\section{Abstract}

As one of the main activities within the state of Rondônia and the country, cattle raising has enormous economic importance. One of the most damaging points for cattle raising is around endoparasitosis in calves in their early life, causing these animals to have a reduction in weight gain. To present the efficacy of the albendazole vermifuge, through 
the analysis of coproparasitology and blood count in Nellore calves was the objective of the present work. Demonstrating in a practical demonstrative way in 30 calves aged 2 to 3 months, of life in a period of 15 days divided equally into: Group I, control, and Group II, treatment, and, finally, stipulate a final average of eggs per gram of feces to evaluate the efficiency of the evermination with $10 \%$ Albendazole orally. Blood counts and coproparasitological tests were performed to show the hematological status of the animals and Eggs per Gram of Feces (OPG). To arrive at the result of effectiveness, the formula used was the calculation of Reduction in Egg Count per Gram of Feces. Thus, the effectiveness of the $10 \%$ Albendazol dewormer in calves between 2 and 3 months was $97.51 \%$. Thus, considered effective, in accordance with the technical regulation of the Common Market Group (GMC). In the hematological context, it was observed on the fifteenth day, a relationship between a greater mean of eosinophils in the hemograms of the animals in the control group (high parasite load), compared to the everted animals (low parasite load).

Keywords: Albendazole; Calves; Coproparasitological; Rondônia.

\section{Resumen}

Como una de las principales actividades dentro del estado de Rondônia y del país, la ganadería tiene una enorme importancia económica. Uno de los puntos más dañinos para la cría de ganado es la endoparasitosis en los terneros en sus primeros años de vida, lo que hace que estos animales tengan una reducción en el aumento de peso. Presentar la eficacia de la vermífuga de albendazol, a través del análisis de coproparasitología y hemograma en terneros Nellore fue el objetivo del presente trabajo. Demostrar de forma práctica demostrativa en 30 terneros de 2 a 3 meses, de vida en un período de 15 días divididos a partes iguales en: Grupo I, control, y Grupo II, tratamiento, y, finalmente, estipular un promedio final de huevos por gramo de heces para evaluar la eficacia de la eternidad con albendazol al $10 \%$ por vía oral. Se realizaron hemogramas y pruebas coproparasitológicas para mostrar el estado hematológico de los animales y huevos por gramo de heces (OPG). Para llegar al resultado de efectividad, la fórmula utilizada fue el cálculo de la Reducción del Conteo de Huevos por Gramo de Heces. Así, la efectividad del antiparasitario Albendazol al 10\% en terneros entre 2 y 3 meses fue del 97,51\%. Por lo tanto, se considera eficaz, de acuerdo con el reglamento técnico del Grupo Mercado Común (GMC). En el contexto hematológico, se observó al decimoquinto día, una relación entre una mayor media de eosinófilos en los hemogramas de los animales del grupo control (alta carga parasitaria), en comparación con los animales evertidos (baja carga parasitaria).

Palabras clave: Albendazol; Pantorrillas; Coproparasitológico; Rondônia.

\section{Introdução}

A pecuária é, sem dúvidas, a principal atividade econômica no estado de Rondônia, sendo o $7^{\circ}$ maior rebanho do país. À vista disso, a Embrapa-RO fez então estimativas de que o rebanho bovino no estado de Rondônia, até 2025, seja de aproximadamente 17.8 milhões de cabeças. Com isso, observa-se tamanha importância da pecuária no setor econômico do estado e do país (Taborda, 2017).

A bovinocultura é a maior fonte de proteína animal consumida, sendo assim, de extrema relevância econômica em todo o país. Contudo, o rendimento e a produção desses animais são afetados de forma constante, em consequência de questões sanitárias, estando os parasitos como os fatores fundamentais dessa queda de produtividade no rebanho bovino em todo o mundo, especialmente nos países tropicais (Cançado, 2012).

O prejuízo do produtor com animais infestados de parasitos, observando apenas o que é considerado "indireto", é de uma queda de $20 \%$ de ganho de peso. Ao considerarmos os demais custos, como a aquisição de produtos antiparasitários, essa perda para o criador aumenta de forma considerável (Cançado, 2012; Gonçalves et al. 2020).

Os bezerros são os mais afetados por parasitos, devido a sua baixa imunidade e imaturidade nos demais mecanismos de defesa, sendo de suma importância um bom manejo sanitário (Caixeta \& Carmo, 2020). Bezerros infectados por parasitas, têm seu desenvolvimento ponderal retardado. Por esse motivo, há quedas na produtividade animal dos rebanhos, sendo um grande problema para os produtores de gado de corte e leite. Parasitos como as coccidioses, podem ocasionar imunossupressão e abrir portas para infecções secundárias. Em casos extremos de infestação parasitária, poderá ocorrer obstruções intestinais, hemorragias e até a morte (Gonçalves, 2000; Petry et al. 2017).

Como definição basal, os parasitos são organismos que se alimentam de outros seres vivos, para sobreviver e/ou reproduzir-se (Cançado, 2012). Estes, diminuem a produtividade animal, devido a sua competição com o hospedeiro pelos 
nutrientes, provindos através da alimentação (Costa, 2011). Entre os principais parasitos dos bovinos, pode-se citar os Helmintos, Coccídeos, Agentes da Tristeza Parasitária Bovina (TPB), e as moscas e carrapatos sendo os principais vetores de algumas destas citadas acima (De Vos, 1992; Hillesheim, 2016; Silva, 2008).

Observa-se que para haver um menor índice de parasitos nos rebanhos bovinos, deve-se fazer everminações em todos os animais, de todas as idades, em especial os animais jovens, pois são mais susceptíveis a ocorrência de parasitoses. Devido a isso, tem-se como base, fazer controles estratégicos dos parasitos e vetores (Conde \& Borges, 2017; Okuda, 2017).

Referente as análises laboratoriais, o hemograma é um meio de avaliação importante uma vez que, juntamente ao exame clínico e outros exames auxiliares, pode fornecer informações relevantes de custo baixo quanto à natureza e evolução do quadro clínico do animal (Rocha, 2013).

A contagem em material fecal, dos ovos de diversos helmintos intestinais é um parâmetro de valor confiável, como índice da intensidade de verminoses e recurso laboratorial de avaliação da eficácia de procedimentos terapêuticos (Castilho, 1984).

Os vermífugos têm uma importância muito grande na pecuária. Esses, são responsáveis pela a prevenção de várias doenças que podem vir ocorrer no rebanho, com isso, mantendo a saúde dos animais. O Albendazol é um vermífugo, de amplo aspecto, pertencente a classe dos benzimidazólicos. É indicado no controle das principais verminoses gastrointestinais e pulmonares, os nematódeos, indicado também para os cestódeos adultos e formas encistadas e para os trematódeos, possuindo ação contra larvas e ovos, tendo ação sobre as três fases de vida dos parasitos (Okuda, 2017).

Em virtude da importância da everminação efetiva dos bovinos, em especial os bezerros, este estudo teve como objetivo avaliar, de forma qualitativa, a eficácia do Sulfóxido de Albendazol à 10\%, via oral, no tratamento de endoparasitos, analisando e confrontando os resultados dos hemogramas e coproparasitológicos (OPG) em bezerros Nelore com idade entre 2 a 3 meses.

\section{Materiais e Métodos}

O trabalho foi realizado em uma fazenda no município de Buritis-RO, Brasil. Foi selecionado um lote de trinta bezerros Nelore com idade entre 2 a 3 meses de vida, de ambos os sexos, que nunca haviam recebido vermífugo. O lote foi divido em dois grupos de 15 animais, de forma igualitária: I - Grupo controle (não tratado); II - Grupo tratamento (tratado) com Albendazol à $10 \%$ via oral na dose de $1,5 \mathrm{ml} / 20 \mathrm{~kg}$. Foi coletado, para as análises, sangue e fezes dos bezerros no primeiro dia (dia 0 ) e décimo quinto dia (dia 15) após o tratamento.

O sangue foi coletado, de ambos os grupos, com agulhas de calibre 40x12, pela veia jugular e armazenados em tubos com EDTA+, de forma individual e identificada. Os tubos permaneceram cerca de quinze minutos em temperatura ambiente e, então, foram acondicionados em caixa térmica com gelo reciclável, para ser efetuado o transporte para o laboratório de Patologia Clínica, onde foram processados os hemogramas através de esfregaços sanguíneos e leitura em máquina de hemograma.

As Fezes foram coletadas, de ambos os grupos, via retal, com luvas de procedimento e, acondicionadas em potes coletores, sendo identificados e colocados em caixa térmica com gelo reciclável, para também serem transportadas para o laboratório de Patologia Clínica. Onde foram realizados os exames de Coproparasitológico, utilizando o princípio de flutuação, através da técnica de Willis-Mollay.

Logo após a coleta de material, foi realizado a everminação do Grupo II, utilizando o vermífugo Albendazol à 10\%, via oral, com base na dose recomendada na bula do produto, de acordo com o fabricante, sendo esta de 1,5 ml / 20 kg. O Grupo I não recebeu a everminação, sendo este o grupo controle. Após o período de quinze (15) dias pós-administração do vermífugo, foi realizado uma nova coleta de material dos dois grupos do lote, verificando e confrontando os resultados do Coproparasitológico, quanto o hemograma (Pereira-Junior, 2017). 
Os animais foram identificados como os animais tratados (grupo II ou tratamento) e, porção inferior, animais não tratados (grupo I ou controle).

As amostras sanguíneas foram coletadas, processadas e analisadas em um período menor que quarenta e oito horas, em ambas as coletas, para que não ocorresse hemólise e, assim, perdendo material essencial para as análises. Estas foram realizadas de forma manual e mecânica, com a produção de lâminas de todos os animais, e a análise através da máquina.

As amostras fecais foram coletadas, processadas e analisadas em um período entre quarenta e oito horas e setenta e duas horas (refrigeradas), para que estas não ficassem comprometidas para análise. Foi utilizada a técnica de Willis-Mollay adaptada para contagem de ovos por grama, que consiste em contar os ovos de helmintos nos quatro bordos da lamínula, multiplicá-los por cinquenta (50) e dividir por quatro ( $X=4 \times 50 \div 4)$.

Com o término do período de análises laboratoriais, foi realizado a avaliação dos hemogramas, comparando prétratamento (dia 0) e pós-tratamento (dia 15) (Pereira-Junior, 2017). Com as amostras fecais examinadas, foi feito o cálculo de Redução da Contagem de Ovos por Gramas de Fezes, de acordo com Buzzulini (2006), para assim, determinar se houve ou não a eficácia na utilização do medicamento (GMC, 1996).

\section{Resultados e Discussão}

O exame de hemograma demonstrou dados semelhantes em ambos os grupos observados no dia zero, tanto em sua série vermelha, quanto em sua série branca. Após a sintetização dos grupos, observá-los e compará-los, foi observada pouca diferença entre os hemogramas do Grupo I para com o Grupo II, como demonstrado nas tabelas a seguir.

A Tabela 1 apresenta a média do Hemograma do grupo I (controle) e grupo II, no dia 28 de outubro e no dia 11 de novembro.

Tabela 1 - Média do Hemograma do grupo I (controle) e grupo II, nos dia 28 de outubro e 11 de novembro, contendo os dados: Série Vermelha; Resultados; Valores de Referência; Série Branca.

\begin{tabular}{cccccc}
\hline \multirow{2}{*}{ Análises } & \multicolumn{3}{c}{ Resultados } & Valores de Referência \\
\cline { 2 - 6 } & \multicolumn{2}{c}{$\mathbf{2 8 / 1 0}$} & \multicolumn{2}{c}{$\mathbf{1 9 / 1 1}$} & \\
\cline { 2 - 6 } Série Vermelha & $\begin{array}{c}\text { Grupo I } \\
\text { (controle) }\end{array}$ & Grupo II & Grupo I (controle) & Grupo II & $5,0-10,0(\mathrm{x} 106 / \mathrm{uL})$ \\
\hline Hemácias & 11.55 & 11.06 & 10.46 & 11.86 & $8-15 \mathrm{~g} / \mathrm{Dl}$ \\
Hemoglobina & 11.66 & 11.18 & 11.7 & 41.76 & $24-46 \%$ \\
Hematócrito & 41.9 & 41.7 & 40.0 & 35.28 & $40-60 \mathrm{Fl}$ \\
V.C.M & 38.85 & 39.1 & 35.8 & 10.04 & $14,4-18,6 \mathrm{Pg}$ \\
H.C.M. & 10.18 & 11.2 & 10.5 & 28.48 & $30-36 \mathrm{~g} / \mathrm{Dl}$
\end{tabular}

\section{Série Branca}

\begin{tabular}{cccccc}
\hline Leucócitos Totais & 22.786 & 24.607 & 24.600 & 22.900 & $4,0-12 \mathrm{Mil} / \mathrm{mm}^{3}$ \\
Neutrófilos & 7.348 & 8.397 & 9.842 & 10.500 & $600-4.000 \mathrm{Mil} / \mathrm{mm}^{3}$ \\
Eosinófilos & 1.311 & 1.029 & 672 & 825 & $0-2.400 \mathrm{Mil} / \mathrm{mm}^{3}$ \\
Linfócitos & 13.484 & 13.158 & 12.557 & 10.500 & $2.500-7.500 \mathrm{Mil} / \mathrm{mm}^{3}$ \\
Monócitos & 1.020 & 1.308 & 1.385 & 1.192 & $24-840 \mathrm{Mil} / \mathrm{mm}^{3}$ \\
Basófilos & - & - & - & - & $0-200 \mathrm{Mil} / \mathrm{mm}^{3}$ \\
Plaquetas & 175.333 & 239.909 & 337.000 & 377.600 & $100.000-800.000 \mathrm{~mm}$ \\
\hline
\end{tabular}


Em todos os hemogramas, na série branca, foi constatada leucocitose por linfocitose, neutrofilia e monocitose, juntamente, todos os animais apresentavam o agente Anaplasma marginale parasitando hemácias, justificando a leucocitose. Sendo os animais tratados para tal enfermidade após o estudo.

$\mathrm{Na}$ leitura dos esfregaços foi observado grande quantidade de hemácias crenadas e pequenas. Efeito que pode ser explicado pela presença da Tristeza Parasitária Bovina e, também explicando o V.C.M, H.C.M e C.H.C.M, abaixo da normalidade.

Confrontando os resultados médios do OPG e os eosinófilos no dia quinze, é possível perceber que o grupo controle ao décimo quinto dia apresentou uma média de eosinófilos maior que o grupo tratamento (controle: $1029 \mathrm{~mm}^{3}$; tratamento: 672 $\mathrm{mm}^{3}$ ), acompanhado de uma parasitose nos animais do grupo controle e baixíssima carga parasitária no grupo tratamento.

O exame coproparasitológico demonstrou considerável carga parasitária em ambos os grupos observados no dia zero (grupo tratamento: 535,8 OPG e grupo controle: 1024 OPG). Após 15 dias da administração de 1,5 ml / 20 kg de Albendazol à $10 \%$ no grupo tratamento, os resultados obtidos foram: grupo tratamento: 13,3 OPG e grupo controle: 518,2 OPG.

Foi confirmada a eficácia do Albendazol através do cálculo de Redução da Contagem de Ovos por Gramas de Fezes (Buzzulini, 2006). Onde o resultado foi de 97,51\%, podendo-se considerar um tratamento efetivo (> 98\% é considerado altamente efetivo; entre 90-98\% é considerado efetivo; 80-89\% é moderadamente efetivo e; <80\% se considera Insuficientemente Ativo).

A Tabela 2 apresenta os dados obtidos através da análise e processamento das fezes, através da técnica de Willis-Mollay.

Tabela 2 - Contagem de ovos por grama de fezes (OPG) e porcentagem de redução de ovos por grama de fezes (RCOF) no dia pré-tratamento (D0) e no dia pós-tratamentos (D15).

\begin{tabular}{cccc}
\hline Grupos & D0 & D15 & RCOF \\
\hline Grupo I (Controle) & 1.024 & 518,2 & $49,4 \%$ \\
Grupo II (Tratamento) & 535,8 & 13,3 & $97,51 \%$ \\
\hline
\end{tabular}

Fonte: Autores.

Araki et al. (2017) demonstraram em seu estudo com bezerros entrando em confinamento que, além de eliminar praticamente todos os endoparasitos do grupo tratado, houve uma diferença significativa em ganho de peso. No estudo supracitado, houveram dois grupos, o controle e o grupo tratado, utilizando Febendazole $5 \mathrm{mg} / \mathrm{kg}$. Como resultado, foi obtido uma eliminação de quase todos os endoparasitos no grupo tratado, bem como o aumento no ganho de peso em 62,3g a mais nos animais do grupo tratado. O presente trabalho visou apenas na averiguação da funcionalidade do albendazol para o tratamento de endoparasitose, o que se mostrou muito efetivo.

Onizuka (2016) realizou em seu trabalho uma avaliação do desenvolvimento ponderal de bovinos precoces tratados com dois tipos de medicamentos antiparasitários, o sulfóxido de albendazole $10 \%$ e a ivermectina, nos meses de julho e setembro. Como resultado, ele observou que os animais tratados com o sulfóxido de albendazole $10 \%$ demonstraram um desenvolvimento ponderal melhor que o achado no grupo controle e no grupo ivermectina. Isso corrobora com o que foi apresentado no atual estudo, onde se obteve uma taxa de porcentagem de redução de ovos por grama de fezes (RCOF) de 97,51\%. Utilizando-se também de ivermectina e albendazol em seu experimento, Neves et al. (2020) descrevem em seu estudo que a animais não são afetados por parasitos nematóides quando são muito bem suplementados com concetrados adequados.

Em seu estudo, Barbosa et al. (2021) utilizaram-se de Doramectina à 1\% (dual oil) para averiguar a sua eficácia no controle de parasitos gastrintestinais em dois grupos distintos, o grupo tratado e o grupo controle. Ao término do trabalho, observaram que o tratamento a base de Doramectina à $1 \%$ se mostrou eficaz em bezerros de corte da raça Angus para o aumento no ganho de peso, mas não foi tão eficaz no controle parasitário. 
Research, Society and Development, v. 10, n. 17, e59101718920, 2021

(CC BY 4.0) | ISSN 2525-3409 | DOI: http://dx.doi.org/10.33448/rsd-v10i17.18920

\section{Conclusão}

Com o término do estudo, pode-se afirmar que a administração de Albendazol à 10\% via oral, em dose única, foi efetiva para combater a endoparasitoses em bezerros, além da eosinofilia encontrada nos hemogramas corroborar com os achados nos coproparasitológicos.

\section{Referências}

Araki, N. A. et al. (2017). Anti-helmínticos na entrada do confinamento de bovinos. Anais Da X Mostra Científica FAMEZ/UFMS. Campo Grande.

Barbosa, A. A. et al. (2021). Efeito da utilização de Doramectina $1 \%$ (dual oil) sob o tratamento anti-helmíntico e ganho de peso de bezerros de corte. Brazilian Journal of Development, 7(3): 32153-32165.

Buzzulini, C. (2006). Eficácia anti-helmíntica comparativa da associação albendazole, levamisole e ivermectina à moxidectina 1\% em ovinos naturalmente infectados por nematódeos gastrointestinais, 2006. 113p. Dissertação (Mestrado em zootecnia) - Faculdade de Ciências Agrárias e Veterinárias, Universidade Estadual Paulista "Júlio Mesquita Filho", Jaboticabal.

Caixeta, D. G. \& Carmo, J. P. (2020). Criação de Bezerros Neonatos: manejo e bem estar. Scientia Generalis, 1(3): 92-103.

Cançado, P. H. D. et al. (2012). Controle parasitário de bovinos de corte em sistemas de integração. Embrapa Gado de Corte.

Conde, M. H. \& Borges, F. A. (2017). Influência dos Protocolos de vermifugação para Ruminantes na Resistência anti-helmíntica. Anais Da X Mostra Científica FAMEZ / UFMS. Campo Grande.

Costa, V. M. (2011). Tristeza parasitária bovina no Sertão da Paraíba. Pesq. Vet. Bras., 31(3): 239-243.

De Vos, A. J. (1992). Distribution, economic importance and control measures for Babesia and Anaplasma. In: WORKSHOP, ILRAD, Nairobi, Kenya, 1991. Proceedings... T.T. Dolan (Editor), 1992. 312 p. 3-15.

Gonçalves, P. M. (2000). Epidemiologia e Controle da Tristeza Parasitária bovina na região sudeste do Brasil. Ciência Rural, $30(1)$ : 187-194.

Gonçalves, R. C., Bono, J. A. M. \& Reis Neto, J. F. (2020). Análise de Dois Sistemas de Produção Pecuária Aplicando um Modelo de Gestão. UNICIÊNCIAS, 24(2): 119-123.

Grupo Mercado Comum (GMC). (1996). Regulamento técnico para registros de produtos antiparasitários de uso veterinário. Decisão N4/93. MERCOSUL, Resolução $N^{o} 76$.

Hillesheim, L. O. \& Freitas, F. L. C. (2016). Ocorrência de Eimeriose em Bezerros criados em propriedades de agricultura Familiar - Nota Científica. Ciência Animal brasileira, 17(13): 472-481.

Neves, J. H., Carvalho, N. \& Amarante, A. F. T. (2020). Gastrointestinal nematode infections do not hinder the development of Simmental X Nellore crossbred calves raised with a nutritionally enhanced diet. Revista Brasileira de Parasitologia Veterinária, 29(1): e015819.

Okuda, L. H. \& APTA (2017). Mortalidade de bezerros - Manejo e Prevenção. Agência Paulista de Tecnologia dos Agronegócios - Governo do estado de São Paulo.

Onizuka, M. K. V. Desenvolvimento ponderal de bovinos mantidos à pasto e em confinamento, submetidos a dois tratamentos endoparasiticidas. Dissertação (Mestrado em Medicina Veterinária - Patologia Clínica), Universidade Estadual Paulista - UNESP, Câmpus de Jaboticabal, São Paulo.

Pereira-Junior, R. A. et al. (2017). Eficácia de Ivermectina e Albendazol contra nematódeos gastrointestinais em rebanho ovino naturalmente infectado no município de Palmas-TO, Brasil. Revista Científica de Medicina Veterinária. 28, Periódico Semestral.

Petry, A. L. et al. (2017). Prevalênia de verminose bovina em propriedades de agricultura familiar no município de Realeza, estado do Paraná, Brasil. Ver. Acad. Ciênc. Anim., 15(2): s557-558.

Rocha, T. G. et al. (2013). Hemograma e proteínas de fase aguda de bezerros sadios do nascimento aos 30 dias de idade. Pesquisa Veterinária Brasileira, 33(1): 25-31.

Silva, F. J. M. (2008). Relação entre infestação natural por Rhipicephalus (Boophilus) microplus (Acari: Ixodidae) e níveis de anticorpos da classe IgG para os agentes da Tristeza Parasitária Bovina e Borrelia sp. em bezerros. 145 f. Dissertação (Mestrado em Ciências Veterinárias) - Instituto de Veterinária, Universidade Federal Rural do Rio de Janeiro, Seropédica.

Taborda, J. M. M. et al. (2015). Impactos sobre o desenvolvimento da pecuária de corte no Estado de Rondônia e projeções de crescimento. Anais do VI encontro de iniciação à pesquisa da Embrapa Rondônia e I Encontro de Pós-graduação. 